\section{Early Career Investigator}

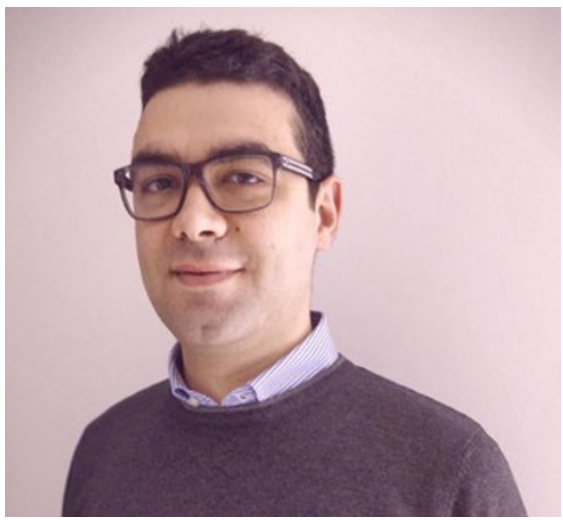

Congratulations to Livio Provenzi, this issue's Early Career Investigator. His Commentary describes his birth in Milan, his work toward obtaining a $\mathrm{PhD}$ in psychology, and his role in the Preterm Behavioral Epigenetics study, which examines the impact of painful and invasive procedures on preterm infants. His career clearly shows the benefits of an international network of mentors! In a study reported in this issue, he and colleagues established normative values for the Neonatal Intensive Care Unit Network Neurobehavioral Scale for infants at 1 month of age. See pages 1087 and 1104

\section{Cord blood inflammation- related proteins in ELGANs are associated with later obesity}

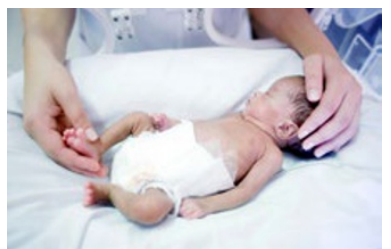

Perrin et al. evaluated 25 inflammatory proteins in extremely-low-gestationalage newborns (ELGANs) in relation to body weight at 2 years of age. They found a positive association for several of the proteins and concluded that inflammation precedes obesity in this cohort. This article is the inspiration for the Policy Commentary this month.

See page 1110

\section{Selection of measures of drug response in pediatrics}

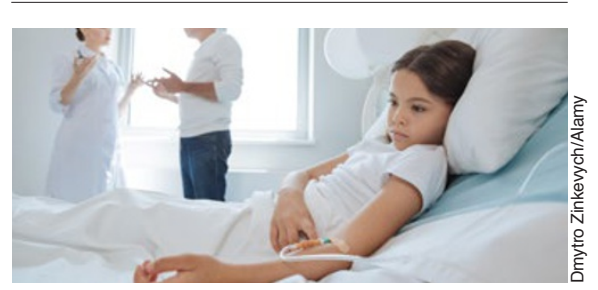

Kelly et al. review the critical methodology for selecting measures of response in pediatric efficacy and safety studies. Among the endpoints discussed are biomarkers, modeling, compliance, and outcome scoring systems, as well as the necessary validation of these pharmacodynamic endpoints. See page 1095

\section{High-dose aspirin for} Kawasaki disease does not improve coronary arterial abnormalities

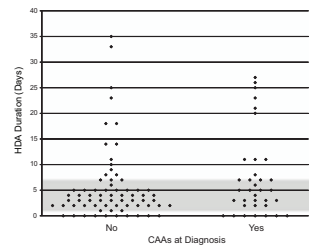

Migally et al. studied 103 patients with newly diagnosed Kawasaki disease who were followed for 9-15 months. High-dose aspirin treatment for either 1-7 days or more than 7 days had no effect on clinically relevant coronary artery outcomes. See page 1136

\section{Obese mothers increase IVH} risk in preterm infants due to lower gestational age at birth

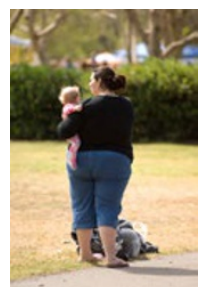

Pai et al. tested the hypothesis that maternal obesity in mothers raises the likelihood of intraventricular hemorrhage (IVH) in preterm offspring by causing increased inflammation. Using a large California database, they found a higher incidence of IVH in preterms born to obese mothers that was nonsignificant when adjusted for gestational age. The authors conclude that maternal obesity increases the risk of preterm birth, which is a known risk factor for IVH. See page 1146

\section{Age-dependent differences in cardiac L-type $\mathrm{Ca}^{2+}$ channels}

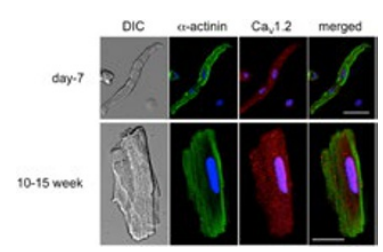

Verapamil is contraindicated in neonates owing to its side effects of hypotension and bradycardia. Sagawa et al. examined the effects of verapamil, nifedipine, and diltiazem on ventricular myocytes obtained from mice at various ages, and found an age dependence only for verapamil. See page 1207

\section{Hypoxic-ischemic injury increases cholesterol turnover}

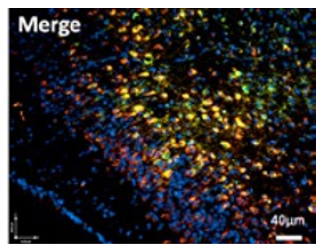

Lu et al. examined cholesterol and its metabolite 24S-hydroxycholesterol in brain and serum and the expression of brain-specific cholesterol 24-hydroxylase using the Vannucci model of hypoxia-ischemia (HI). They found enhanced cholesterol metabolism early after $\mathrm{HI}$, and postulate that 24S-hydroxycholesterol may be a biomarker for Hl injury. See page 1218 